\title{
Static Strength of Circular Tubular T-joints with Inner Doubler Plate Reinforcement Subjected to Axial Compression
}

\author{
HE Shubin, Shao Yongbo* and Zhang Hongyan
}

\author{
School of Civil Engineering, Yantai University, Qingquan Road 32\#, Laishan District, Yantai, Shandong Province, PR \\ China 264005
}

\begin{abstract}
For an inner doubler plate reinforced tubular joint, the doubler plate is welded onto the inner surface of the chord by using fillet weld, and the position of the doubler plate is placed under the brace/chord intersection. Through this reinforcement, the radial stiffness of the chord is improved, and hence the strength is increased. Finite element model is built by using software ABAQUS to analyze the ultimate load and failure mode of inner doubler plate reinforced tubular T-joints. Overall 432 models with different geometrical parameters and doubler plate sizes are analyzed. The effect of all the parameters on improving the static strength is discussed. Finally, a parametric equation for predicting the static strength of a tubular T-joint with inner doubler plate is presented through curve fitting technique, and its accuracy and reliability are also evaluated by error analysis.
\end{abstract}

Keywords: Circular tubular T-joint, inner double plate, static strength, finite element analysis, parametric equation

\section{INTRODUCTION}

Tubular structures with hollow section are used widely in offshore engineering due to its advantages such as light weight, high strength and low friction coefficient to wind and seawater. In tubular structures, brace member is welded onto the surface of chord member to form brace/chord connection which is also called tubular joint. Welded tubular structures behave very like truss structure, in which each tube member is mainly subjected to axial loading. Such mechanism makes the chord member be subjected loading in its radial direction. As it is known that the radial stiffness of a hollow section tube is much smaller than its axial stiffness, failure thus mostly occurs at the surface of the chord near the brace/chord intersection. Therefore, most reinforcing methods for tubular joints are to improve the radial stiffness of the chord member.

In the reported reinforcing methods, two types can be classified due to the location of the reinforcement: outer reinforcement and inner reinforcement. Doubler plate reinforcement, collar-plate reinforcement and bracket reinforcement are the typical outer reinforcing methods, and there are many research studies in this field [1-5]. Accordingly, internally stiffened ring reinforcement, inner plate reinforcement and local chord reinforcement are some representative inner reinforcing methods. Refs. [6-10] studied tubular joints with internally stiffened ring reinforcement through experimental investigation and finite element analysis. The results show that internally stiffened ring can improve the static strength

*Address correspondence to this author at the School of Civil Engineering, Yantai University, Qingquan Road 32\#, Laishan District, Yantai, Shandong Province, PR China 264005; Tel: +86 535 6902606; Fax: +86 535 690255; E-mail: cybshao@ytu.edu.cn of tubular joints efficiently. The inner plate reinforcement on tubular joints is introduced in Refs. [11-13], and it is also found this reinforcing method has also efficiency in increasing the load bearing capacity of tubular joints. For local chord reinforcement, Ref. [14] provided experimental study and found an effective reinforced result can also be obtained. Compared with outer reinforcing method, inner reinforcing method does not affect the appearance since all the reinforcements are placed inside the chord member. It is hence used by most designers in practice.

Inner doubler plate reinforcement is a similar inner reinforcing method compared with other inner reinforcing methods. However, its study has not been carried out although it has been used in practical design. Due to this reason, a detailed study and discuss will be conducted in this paper. However, it should be stressed here that the doubler plate is practically placed inside the chord tube only when the chord diameter is relatively bigger because the welding process can be operated in this case. When the chord diameter is smaller, the doubler plate should be placed outside on the chord surface although it is better to place the doubler plate inside the chord to keep the appearance of the entire structure.

There are several types of joints used in welded tubular structures in practice. For brevity, a simple tubular T-joint is selected to study. Based on this study, similar conclusion can be obtained in assessing the reinforcing efficiency. In addition, when a tube consisting of a tubular joint has big slenderness, axial load is dominant in all the loading cases. Therefore, only axial load is considered hereby. As a tubular joint has bigger load carrying capacity in tension than in compression, only compression is selected as the loading case to the tubular joint. 


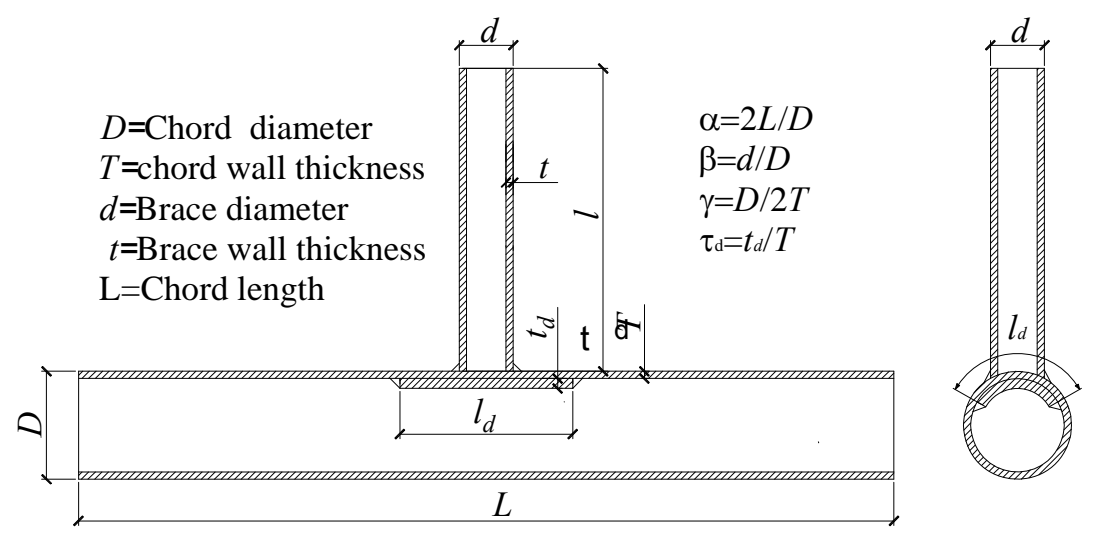

Fig. (1). Geometry model of circular tubular joint with inner doubler plate.

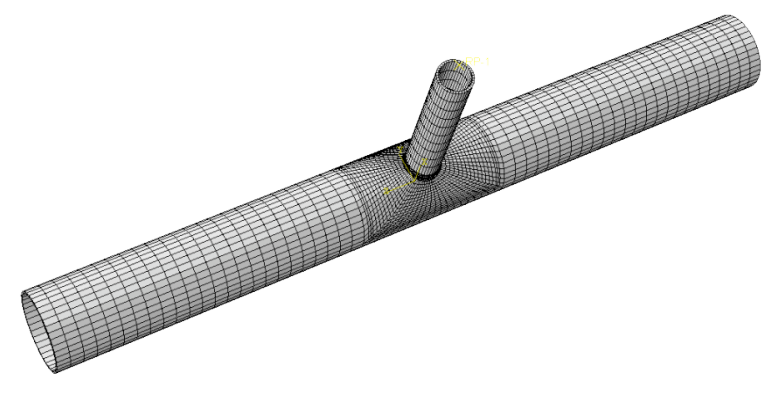

Fig. (2). Finite element model of reinforced T-joint.

\section{FINITE ELEMENT MODEL}

\section{Geometrical Model of an Inner Doubler Plate Reinforced Tubular Joint}

The configuration of a tubular T-joint with inner doubler plate reinforcement is shown in Fig. (1). The doubler plate is placed inside the inner surface of the chord, and it is connected to the chord through fillet welding. To ensure full contact, the doubler plate is bent to a curved plate with same curvature as the chord before welding. The position of the doubler plate is just under the brace/chord intersection because this location is generally the failure position. For constructional brevity, the doubler plate can be designed as rectangular or square shape. Fig. (1) shows a square doubler plate, and this shape is also used in the finite element analysis in the following sections. It is assumed all the quantities are denoted in upper case letter for chord member and the lower case letter is used to denote the quantities for brace member. Lower case letter with subscript "d" is used to denote the corresponding quantities of the doubler plate. Some normalized parameters for describing the geometry of a tubular joint are also provided in Fig. (1).

\section{Finite Element Model}

To build an accurate and reliable finite element model, a high quality mesh is necessary as well as reasonable definition on material properties, loading application and boundary conditions. A high quality mesh is obtained from reasonable element selection, mesh discretizing scheme and good element shape. In addition, refined mesh should be taken in high stress gradient region while relatively coarse mesh can

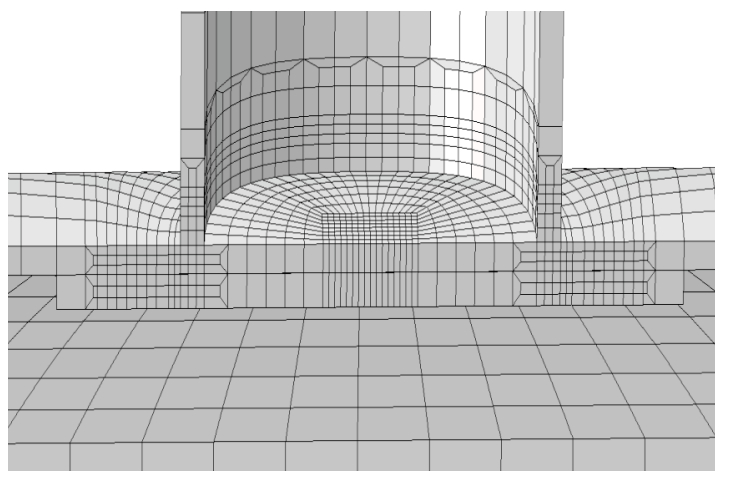

be used in the region far away from critical positions for saving computing time. For a tubular joint, high stress gradient region is located at the brace/chord intersection because high stress concentration exists here. Fig. (2) shows the mesh of an inner doubler plate reinforced tubular T-joint. Three layers of elements are used in tube thickness direction around the intersection while only one layer of elements is used in the other region. C3D8I element is used to generate the mesh because this type of element is advantageous in defining contact problem. For the material properties, elastic-perfect plastic model is used in the analysis and the hardening process of the steel material is ignored. In the finite element analysis, the yield stress of steel material is 235 $\mathrm{N} / \mathrm{mm}^{2}$, and elastic modulus and Poisson's ratio are 206,000 $\mathrm{N} / \mathrm{mm}^{2}$ and 0.3 respectively.

As contact problem exists between the inner doubler plate and the chord inner surface, a definition on contact must be made. Surface-to-surface contact is used in this study, and hard contact in normal direction is defined between two contacting surfaces. In tangential direction, nofriction is used. AS the doubler plate is bent to the same curvature with the chord inner surface, the gap between the doubler plate and the inner surface of the chord is much smaller. A gap less than $1.0 \mathrm{~mm}$ is assumed in the finite element analysis. For the boundary conditions, it is assumed that there is no initial chord stress. In this condition, one end of the chord is pinned while the other end is simulated as a roller. Displacement controlling method is used in the loading process. Only vertical displacement in brace axial direction is applied step by step in such loading process. 
Table 1. Geometric Dimensions of Unreinforced Tubular

\begin{tabular}{|c|c|c|c|c|c|c|}
\hline Specimen & $\begin{array}{c}\boldsymbol{D} \\
(\mathbf{m m})\end{array}$ & $\begin{array}{c}\boldsymbol{d} \\
(\mathbf{m m})\end{array}$ & $\begin{array}{c}\boldsymbol{T} \\
(\mathbf{m m})\end{array}$ & $\begin{array}{c}\boldsymbol{T} \\
(\mathbf{m m})\end{array}$ & $\boldsymbol{\beta}$ \\
\hline \hline $\mathrm{T} 1$ & 203 & 50 & 6 & 6 & 0.246 & 16.91 \\
\hline $\mathrm{T} 2$ & 203 & 90 & 6 & 6 & 0.443 & 16.91 \\
\hline $\mathrm{T} 3$ & 180 & 50 & 8 & 6 & 0.278 & 11.25 \\
\hline
\end{tabular}

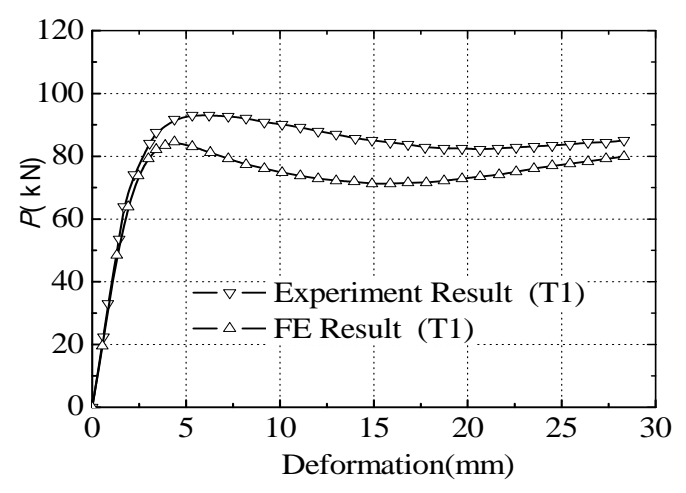

(a)Specimen T1

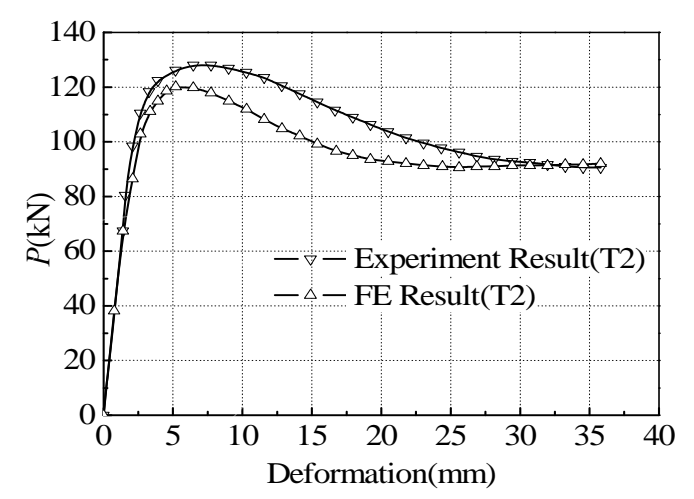

(b)Specimen T2

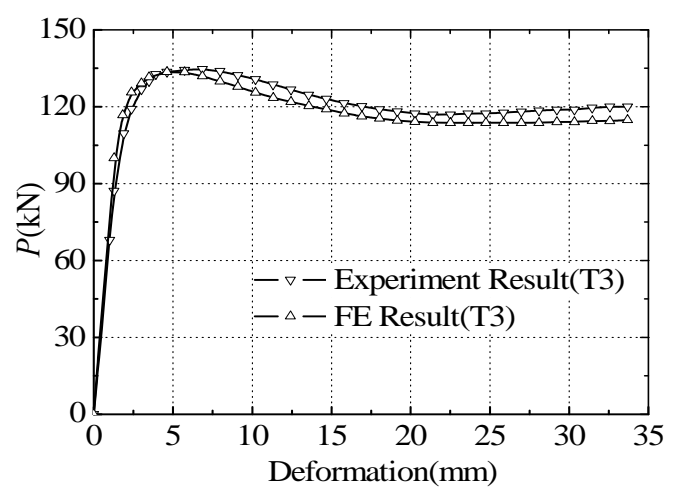

(c)SpecimenT3

Fig. (3). Comparison of load-deformation curve between experiment and finite element model.

\section{Verification of Finite Element Model}

To verify the presented finite element model, some experimental results for un-reinforced tubular T-joints reported in Ref. [15] are used to assess the above modeling method.
Overall 3 T-joint specimens, as tabulated in Table 1, were tested. Other details can be found in Ref. [15].

The load-deformation curves of the 3 specimens are plotted in Fig. (3). The deformation is defined as the top and 
Table 2. Comparison Bearing Capacity between Experiment and Finite Element Model

\begin{tabular}{|c|c|c|c|}
\hline Specimen & Experimental Result $\boldsymbol{P e}(\mathbf{k N})$ & Finite Element Result $\boldsymbol{P}_{\boldsymbol{f}}(\mathbf{k N})$ & Error $\left|\boldsymbol{P e}-\boldsymbol{P}_{\boldsymbol{f}}\right| / \boldsymbol{P e}(\%)$ \\
\hline \hline T1 & 93.04 & 84.57 & 9.10 \\
\hline T2 & 128.02 & 120.16 & 6.56 \\
\hline T3 & 134.72 & 133.54 & 1.88 \\
\hline
\end{tabular}

Table 3. Value of Parameter

\begin{tabular}{|c|c|c|c|c|}
\hline $\boldsymbol{\beta}$ & $\boldsymbol{\gamma}$ & $\boldsymbol{t}_{\boldsymbol{0}}$ & $\boldsymbol{\tau}_{\boldsymbol{d}}$ & $\boldsymbol{l}_{\boldsymbol{d}} / \boldsymbol{d}$ \\
\hline \hline $0.25,0.5,0.7$ & $16,22,28$ & $4,8,12$ & $0.8,1.2,1.6,2.0$ & $1.5,1.75,2.0,2.25$ \\
\hline \hline
\end{tabular}

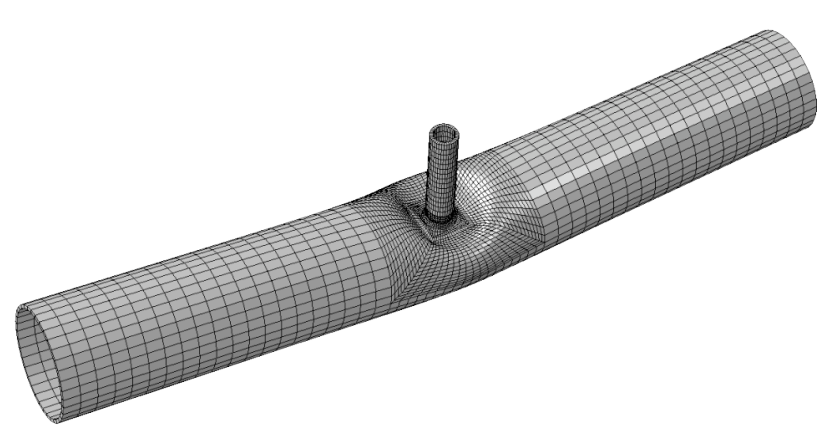

(a) Local Buckling

Fig. (4). Different failure modes for reinforced T-joint.

bottom displacement difference of the chord at mid-span. The peak value of the load in the curves is defined as the static strength. Fig. (3) shows that the finite element results agree reasonably well with experimental measurements. The maximum value of the difference between the two results is less than $10 \%$. Table 2 lists the detailed difference between the two results for the 3 specimens. From the comparison, it is found that the presented finite element model can be used for analyzing the static strength of tubular joints reliably.

\section{PARAMETRIC STUDY}

\section{Values of Parameters in Parametric Study}

To study the effect of different parameters on the static strength of tubular $\mathrm{T}$-joints with inner doubler plate reinforcement under axial compression, a parametric study has been carried out. Overall 5 parameters are considered in the parametric study, which include $\beta, \gamma, t_{0}, \tau_{d}$ and $l_{d} / d$. The values of each parameter in parametric study are provided in Table 3.

Additionally, the value of parameter a $(2 L / D)$ is fixed to be 15 , the brace length is fixed to be 4 times of brace diameter $(l=4 d)$, and the value of $\tau$ is fixed to be 1.0 in parametric analysis.

\section{Failure Mode}

From parametric study, it is found that the failure mode of reinforced tubular T-joints is different when the values of parameters $\beta, \tau_{b}$ and $l_{d} / d$ are different. Fig. (4). shows two typical failure modes of the reinforced T-joint models: local buckling (shown in Fig. (4a) and local yielding (shown in

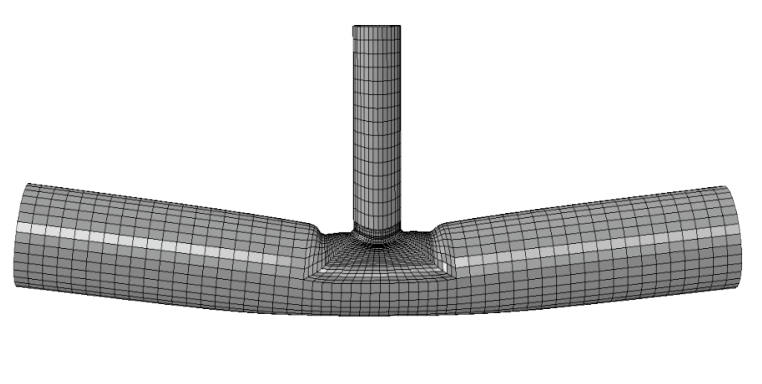

(b) Local Yielding

Fig. (4b). The failure mode is influenced by the reinforcement of inner doubler plate. To explain such effect in details, Figs. (5a-5f) illustrate different failure modes.

Local buckling occurs at the connection of the doubler plate and the chord when the values of $\beta$ and $l_{d} / d$ are both very small, as shown in Fig. (5a). The deformation of the doubler plate is quite small, and small separation between doubler plate and chord inner surface occurs. In case that the value of $\beta$ is very small while the value of $l_{d} / d$ is bigger, as shown in Fig. (5c), deformation of the doubler plate becomes bigger. This means the reinforcing efficiency is influenced by the size of the doubler plate when the value of parameter $\beta$ is very small.

When the value of $\beta$ is quite big, as shown in Figs. (5d5f), the value of $l_{d} / d$ almost does not affect the failure mode because local yielding occurs in this case and the stiffness of the doubler plate is big enough to resist the radial deformation of the local chord near the brace/chord intersection. It is not efficient to improve the static strength by increasing the size of the doubler plate in this case.

\section{Effect of $\tau_{d}$ and $l_{d} / d$ on Static Strength}

In the parametric study, overall $432 \mathrm{~T}$-joint models with different geometrical parameters and sizes of doubler plate are analyzed. For comparison, the corresponding unreinforced $\mathrm{T}$-joint models are also analyzed. The static strengths of the un-reinforced and the reinforced $\mathrm{T}$-joints under axial compression are denoted by $N_{c r, 0}$ and $N_{c r}$ respectively. Figs. (6a-6c) show the effect of $\tau_{d}$ and $l_{d} / d$ on the static strength with different values of $\beta$. 


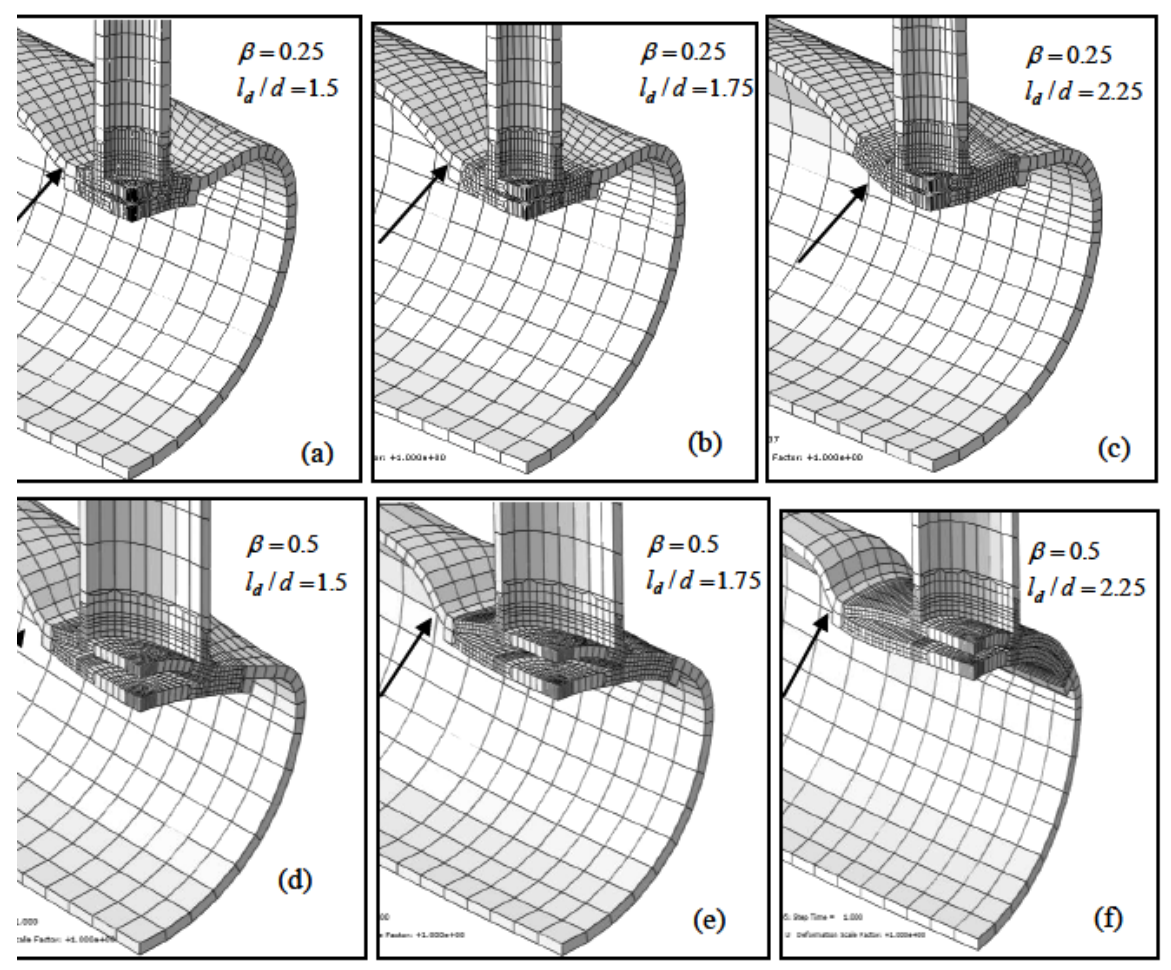

Fig. (5). Deformed shapes of reinforced tubular with $\tau_{d}=1.2$.

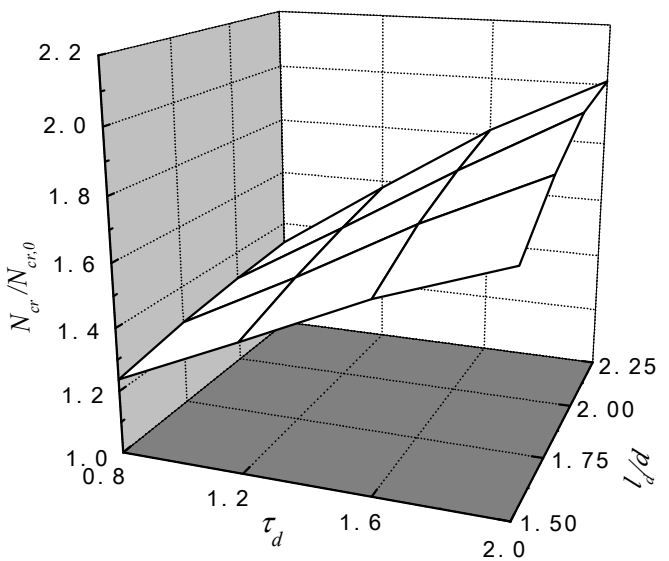

(a) $\beta=0.3$

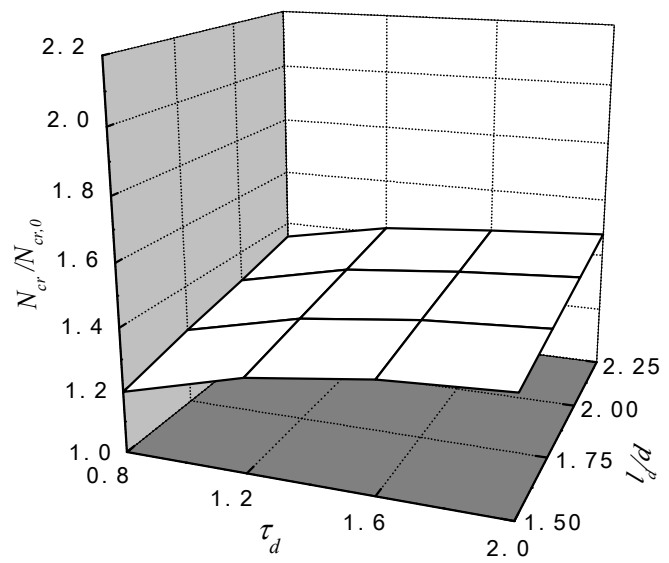




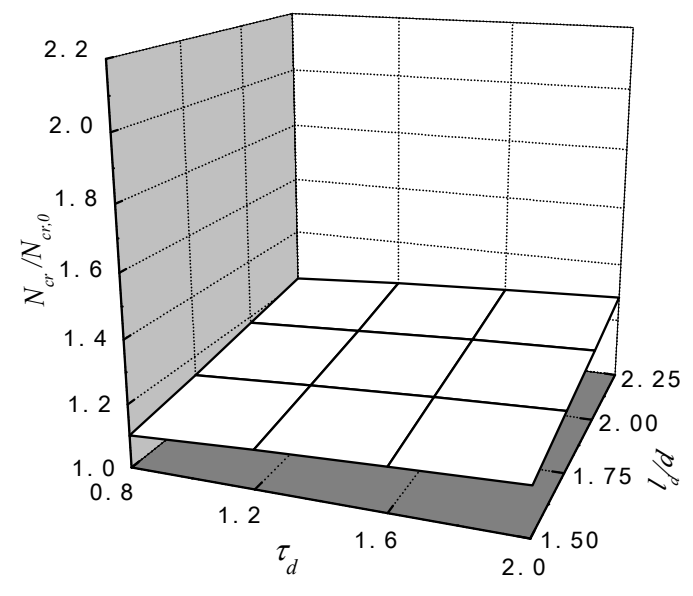

(c) $\beta=0.7$

Fig. (6). Effects of $\tau_{d}$ and $l_{d} / d_{1}$ on static strength with different values of $\beta$.

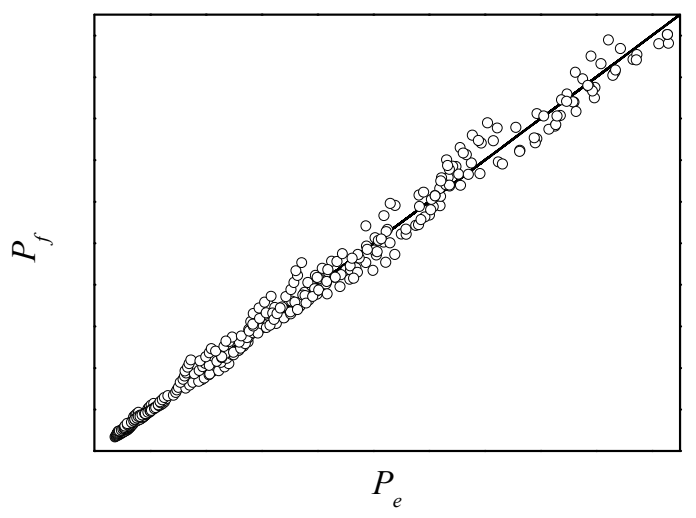

Fig. (7). Comparison between theoretical and numerical results.

When the value of $\beta$ is very small, as shown in Fig. (6a), the effects of both $\tau_{d}$ and $l_{d} / d$ have an obvious effect on the static strength, and such effect is almost in linear. With the increase of $\beta$, the effect of the two parameters become more gradual, as shown in Fig. (6b). Especially when the value of $\beta$ is 0.7 , as shown in Fig. (6c), $\tau_{d}$ and $l_{d} / d$ has very minor effect on improvement of the static strength.

\section{Parametric Equation}

Based on the parametric study and the effect of geometrical parameters and size of doubler plate on the static strength of reinforced T-joints, a parametric equation for predicting the ultimate load of a tubular T-joint with inner doubler plate reinforcement is:

$$
N_{c r}=k N_{c r, 0}
$$

In Eq. (1), the value of $\mathrm{k}$ is determined by parameters $\beta$, $\tau_{d}, l_{d} / d, \gamma$, and it is expressed as follow:

$$
k=\gamma^{*} \tau_{d}^{*} \mu
$$

Where:

$$
\gamma^{*}=8.7055 \gamma^{0.4410}
$$

$$
\begin{aligned}
& \tau_{d}^{*}=\frac{0.6483 \tau_{d}}{19.9693 \beta+3.6338}+1.1809 \\
& \mu=\frac{0.2228 l_{d} / d}{1.5969 \beta+6.3294}+0.1311
\end{aligned}
$$

The static strength of the un-reinforced tubular T-joints under axial compression $N_{c r, 0}$ can be calculated from many parametric equations in many design guidelines in the world. In Chinese Code, $N_{c r, 0}$ is calculated from following equation

$$
N_{c r, 0}=11.51\left(\frac{D}{T}\right)^{0.2} \psi_{n} \psi_{d} T^{2} f_{y}
$$

Where $\Psi_{n}$ is a parameter considering the initial chord stress, $\Psi_{d}$ is a parameter determined by $\beta$ (when $\beta \leq 0.7, \Psi_{d}$ $=0.069+0.93 \beta$; when $\left.\beta \leq 0.7, \Psi_{d}=2 \beta-0.68\right)$.

\section{Assessment of Parametric Equation}

To assess the accuracy of Eq. (1) in calculating the static strength, the results obtained from this parametric equation are compared with finite element results. The comparison is shown in Fig. (7). $P_{e}$ and $P_{f}$ denote the theoretical results calculated from parametric equation and the numerical results obtained from finite element analysis respectively. It can be seen from Fig. (7) that the two results for most models agree quite well. 
To do a detailed investigation, the relative error $e_{i}^{*}$, the average relative error $e$, relative standard deviation $s^{*}$ and average relative standard deviation $s$ are defined as follows

$e_{i}^{*}=\frac{P_{f i}-P_{e i}}{P_{f i}}(i=1,2,3 \ldots \mathrm{n})$

$e=\frac{\sum_{i=1}^{n}\left|e_{i}^{*}\right|}{n}$

$s^{*}=\sqrt{\frac{\sum_{i=1}^{n}\left|e_{i}^{*}\right|^{2}}{n-1}}$

$s=\sqrt{\frac{\sum_{i=1}^{n}\left(\left|e_{i}^{*}\right|-e\right)^{2}}{n-1}}$

where $P_{f i}$ and $P_{e i}$ denote the finite element result and the theoretical result of model $i ; n$ is the total model number.

The values of the above 4 errors obtained from Eqs. (7)(10) are $e=5.71 \%, s^{*}=7.16 \%, \mathrm{~s}=4.32 \%$ respectively. Such values indicate that the proposed parametric equation can produce accurate and reliable estimation for the static strength of a tubular $\mathrm{T}$-joint with inner doubler plate reinforcement.

It is stressed that the validity range of the parametric equation is below:

$$
0.25 \leq \beta \leq 0.7,0.8 \leq \tau_{d} \leq 2.0,1.5 \leq l_{d} / d \leq 2.25
$$

\section{CONCLUSIONS}

Based on the finite element analysis for static strength of tubular T-joints with doubler plate reinforcement, the effect of the doubler plate size on the static strength is studied. A parametric equation for predicting the static strength is also presented. The following conclusions can be obtained:

Inner doubler plate can improve the static strength of tubular T-joints under axial compression effectively in a validity range.

The failure mode of the reinforced T-joints is mainly influenced by parameter $\beta$. A smaller value of $\beta$ can make local buckling occur more easily, and the improvement on the static strength more effective. The bigger value of $\beta$ makes local yielding occurs more easily, and the improvement on the static strength is more ineffective.

The presented parametric equation can produce reasonably accurate estimation for the static strength of a tubular Tjoint with inner doubler plate reinforcement.

\section{CONFLICT OF INTEREST}

The authors confirm that this article content has no conflicts of interest.

\section{ACKNOWLEDGEMENTS}

This study is supported by National Natural Science Foundation (No. 50808153). Such support is appreciated by the authors.

\section{REFERENCES}

[1] G. J. van der Vegte, Y. S. Choo, J. X. Liang, N. Zettlemoyer and J. Y. R. Liew, "Static strength of T-joints reinforced with doubler or collar plates, II: numerical simulations", J. Struct. Eng., ASCE, vol. 131, pp. 129-139, January 2005.

[2] Y. S. Choo, "Recent development and innovation in tubular structures", Adv. Struct. Eng., vol. 8, pp. 217-230, August 2005.

[3] Y. S. Choo, G. J. van der Vegte, N. Zettlemoyer and B. H. Li, "Static strength of T-joints reinforced with doubler or collar platespart I: experimental investigations", J. Struct. Eng., ASCE, vol. 131, pp. 119-128, January 2005.

[4] Y. S. Choo, J. X. Liang, G. J. van der Vegte and J. Y. R. Liew, "Static strength of collar plate reinforced CHS X-joints loaded by in-plane bending", J. Constr. Steel Res., vol. 60, pp. 1754-1760, December 2004

[5] Y. S. Choo, J. X. Liang, G. J. van der Vegte and J. Y. R. Liew, "Static strength of doubler plate reinforced CHS X-joints loaded by in-plane bending", J. Constr. Steel Res., vol. 60, pp. 1725-1744, December 2004.

[6] T. S. Thandavamoorthy, A. G. Madhava Rao and A. R. Santhakumar, "Behavior of internally ring-stiffened joints of offshore platforms" J. Struct. Eng., ASCE, vol. 125, pp. 1348-1352, November 1999.

[7] M. M. K. Lee and A. Llewelyn-Parry, "Offshore tubular T-joints reinforced with internal plain annular ring stiffeners" J. Struct. Eng., ASCE, vol. 130, pp. 942-951, June 2004.

[8] M. M. K. Lee and A. Llewelyn-Parry, "Strength prediction for ring-stiffened DT-joints in offshore jacket structures", J. Struct. Eng., ASCE, vol. 27, pp. 421-430, Feburary 2005.

[9] X. L. Wang, Q. Li and Z. Z. Yin, "Analysis of bearing capacity and deformation of intersecting joints of ring-stiffened circular tubes", J. Lanzhou Univ. Technol., vol. 32, pp. 128-132, May 2006.

[10] F. Zhang, Y. J. Chen, Y. Y. Chen, Y. R. Lin and N. L. Yao, "Effects of ring-stiffeners on the behaviour steel tubular joints", $J$ Space Struct., vol. 10, pp. 51-56, January 2004.

[11] G. Wang and J. C. Zhang, "Study on application of IGP-Stiffen CHS joints in Guang dong Science Center", J. Indust. Constr., vol. 37, pp. 93-97, October 2007

[12] Y. B. Shao, J. C. Zhang, Z. H. Qiu and J. J. Shang, "Strength analysis of large-scale multiplanar tubular joints with inner-plate reinforcement", Int. J. Space Struct., vol. 24, pp. 161-177, September 2009 .

[13] T. Li, Y. B. Shao and J. C. Zhang, "Study on static strength of tubular joints reinforced with horizontal inner plate", J. Steel Constr., vol. 24, pp. 25-29, August 2009.

[14] Y. B. Shao, "Study on reinforcing methods for welded tubular joints structures", J. Yantai Univ., vol. 22, pp. 312-320, December 2009.

[15] Y. Q. Cai, Y. B. Shao and Y. S. Yue, "Experimental Study on Bearing Capacity for Circular Tubular T-joints with Collar Plate Reinforcement", Eng. Mech., vol. 28, pp. 90-95, September 2011. 